\title{
BOUNDARY BEHAVIOUR OF LEVEL CURVES OF HARMONIC FUNCTIONS
}

\author{
W. J. WALKER
}

\begin{abstract}
Sufficient conditions are obtained for a function harmonic in the disk and continuous at a boundary point to have a level curve emanating from the boundary point into the disk. The one-sided derivative of the level curve is studied at the boundary point.
\end{abstract}

The objective of this paper is to answer the following general questions. When does a function which is harmonic in the interior of the unit disk and which has a continuous extension to certain boundary points also have level curves which emanate from these boundary points into the interior of the disk? In particular, how can the implicit function theorem of real variable analysis be used to answer this question?

This approach leads to an unanswered question. Namely, to show the existence of a function which is harmonic in the disk and which has a level curve which emanates from a boundary point of the disk, but in such a way that it does not have a one-sided derivative at this boundary point.

To make these statements precise define $u\left(r e^{i \theta}\right)$ to be harmonic in the open unit disk $U$ and continuous at a point $e^{i \theta_{0}}$, denoted by $\theta_{0}$ on the boundary of $U$. We seek sufficient conditions for the existence of a level curve $\theta=F(r)$ emanating from $\theta_{0}$ into $U$ and corresponding to points $r e^{i \theta}$ where $u\left(r e^{i \theta}\right)=u\left(\theta_{0}\right)$. The left derivative of $F$ at $r=1$ will be denoted by $F_{L}^{\prime}(1)$. The first theorem gives sufficient conditions for the existence of the level curve for $u$. The same conditions are assumed for $v$, the harmonic conjugate of $u$, and these are used to prove the existence of $F_{L}^{\prime}(1)$ for the level curve of $u$.

THEOREM 1. Let $f\left(r e^{i \theta}\right)=u\left(r e^{i \theta}\right)+i v\left(r e^{i \theta}\right)$ be analytic in $U$ and belong to the Hardy space $H^{1}$ so that it is the Poisson integral of an integrable function $f(\theta)=$ $u(\theta)+i v(\theta)$. It is also assumed that $f$ has a continuous derivative $u^{\prime}(\theta)+i v^{\prime}(\theta)$ on an open arc $I$. If $\theta_{0}$ is any point in I at which $u^{\prime}\left(\theta_{0}\right) \neq 0$ then there exists a level curve $\theta=F(r)$ of $u$ which emanates from $\theta_{0}$ into $U$ and the left derivative $F_{L}^{\prime}(1)$ exists. (By symmetry a similar statement holds for $v$.)

Proof. The continuity of $u(\theta)$ and the Poisson integral representation ensure that $u\left(r e^{i \theta}\right)$ is continuous at each point of $I$ (see $[1$, p. 152]). The next step is to prove the continuity of $\partial u / \partial \theta$. This follows from the continuity of $u^{\prime}(\theta)$ by applying a theorem of Fatou to each point of $I$ (see the lemma in [1, p. 155]). We emphasise that this particular lemma holds for any path tending to the boundary point whereas the more well-known theorems of Fatou are stated for nontangential paths.

Received by the editors June 15, 1983.

1980 Mathematics Subject Classification. Primary 31A20; Secondary 42A50.

(C) 1984 American Mathematical Society $0002-9939 / 84 \$ 1.00+\$ .25$ per page 
Since $\partial u / \partial \theta$ is continuous in a neighbourhood of $I$ and since $(\partial u / \partial \theta)\left(e^{i \theta_{0}}\right)=$ $u^{\prime}\left(\theta_{0}\right) \neq 0$, the implicit function theorem (a one-sided version) guarantees the existence of a continuous arc $\theta=F(r)$, representing a level curve emanating from $\theta_{0}$ into $U$.

In order to prove the existence of $F_{L}^{\prime}(1)$ it suffices to show that $(\partial u / \partial r)\left(r e^{i \theta}\right)$ is continuous in a neighbourhood of $I$. By the argument above $\partial v / \partial \theta$ has this property and we can use the Cauchy-Riemann equation $\partial u / \partial r=r^{-1} \partial v / \partial \theta$ in $U$. This shows that $\lim _{r \uparrow 1}(\partial u / \partial r)\left(r e^{i \theta}\right)$ exists. The mean value theorem of real analysis may then be applied for each fixed $\theta$ in $I$ to show that

$$
\frac{\partial u}{\partial r_{L}}\left(e^{i \theta}\right)=\lim _{r \uparrow 1} \frac{\partial u}{\partial r}\left(r e^{i \theta}\right) .
$$

Hence $\partial u / \partial r$ is continuous in a neighbourhood of $I$ and the existence of $F_{L}^{\prime}(1)$ is established.

If it is assumed that $u^{\prime}(\theta)$ is continuous on $I$ and no further smoothness assumptions are made concerning $u$ or $v$, then a level curve $\theta=F(r)$ will be defined emanating from $\theta_{0}$ but it seems possible that $F_{L}^{\prime}(1)$ does not exist. In Theorem 2 stronger assumptions are made about $u^{\prime}(\theta)$.

THEOREM 2. Let $u\left(r e^{i \theta}\right)$ be harmonic in $U$ and continuous on $\bar{U}$ and suppose that $u(\theta)$ is absolutely continuous on the unit circle. If $u^{\prime}(\theta)$ is Dini continuous on an open arc $I$ and $\theta_{0}$ is an interior point of $I$ with $u^{\prime}\left(\theta_{0}\right) \neq 0$, then there exists a level curve $\theta=F(r)$ of $u$ which emanates from $\theta_{0}$ into $U$ and the left derivative $F_{L}^{\prime}(1)$ exists. If $v$ is the harmonic conjugate of $u$ and $v^{\prime}\left(\theta_{0}\right) \neq 0$ then a similar statement holds for the level curve of $v$.

Proof. Since $u$ is continuous on $\bar{U}, u\left(r e^{i \theta}\right)$ has the Poisson integral representation

$$
u\left(r e^{i \theta}\right)=\frac{1}{2 \pi} \int_{0}^{2 \pi} u(\phi) P(r, \phi-\theta) d \phi,
$$

where $P(r, \phi-\theta)$ is the Poisson kernel. Differentiating with respect to $\theta$ under the integral sign, we obtain

$$
\frac{\partial u}{\partial \theta}\left(r e^{i \theta}\right)=-\frac{1}{2 \pi} \int_{0}^{2 \pi} u(\phi) \frac{\partial P}{\partial \phi}(r, \phi-\theta) d \phi
$$

Then by integration by parts and the absolute continuity of $u$ we have the Poisson integral representation

$$
\frac{\partial u}{\partial \theta}\left(r e^{i \theta}\right)=\frac{1}{2 \pi} \int_{0}^{2 \pi} u^{\prime}(\phi) P(r, \phi-\theta) d \phi .
$$

The integrability of $u^{\prime}$ guarantees that the conjugate of $\partial u / \partial \theta$, which is $\partial v / \partial \theta$, can be extended to the boundary almost everywhere by taking nontangential limits (see [2, p. 103]). We now show the continuity of $\partial v / \partial \theta$ on a neighbourhood of $I$. Initially we denote the boundary value of $\partial v / \partial \theta$ on $I$ by $g(\theta)$. The hypothesis that $u^{\prime}(\theta)$ is integrable and Dini continuous on $I$ ensures that $g(\theta)$ is continuous on $I$ (see [2, p. 107]). Similarly $v(\theta)$ is continuous on $I$. If $J$ is any closed subinterval of $I$ then $v\left(r e^{i \theta}\right)$ converges uniformly to $v(\theta)$ on $J$ and $(\partial v / \partial \theta)\left(r e^{i \theta}\right)$ converges uniformly to $g(\theta)$ on $J$. Hence $v^{\prime}(\theta)=g(\theta)$ and $\partial v / \partial \theta$ is continuous in a neighbourhood of $I$. The conclusion of Theorem 2 now follows from Theorem 1. 
Comments. (i) As mentioned earlier, if it is assumed that $u^{\prime}(\theta)$ is continuous on $I$ but no further smoothness assumptions are made concerning $u$ or $v$, then a level curve $\theta=F(r)$ will be defined emanating from $\theta_{0}$, but it seems possible that $F_{L}^{\prime}(1)$ does not exist. Indeed if $\Gamma$ is an analytic curve with this property, which divides $U$ into two components, and $w=f(z)$ maps one of these components conformally on $\operatorname{Re} w>0$, then the function $u(z)=\operatorname{Re} f(z)$ is harmonic in a neighbourhood of $\Gamma$ and vanishes on $\Gamma$. It seems that a different technique will be required to obtain such a curve as the level curve of a function harmonic in the whole disk.

(ii) If $f=u+i v$ is an analytic univalent function on $U$ and $f(U)$ is bounded by a Dini-smooth Jordan curve, then $f^{\prime}(z)$ has a continuous extension to $\bar{U}$ (see $[3, \mathrm{p}$. 298]). In this case Theorem 1 applies to the boundary of $U$.

\section{REFERENCES}

1. N. K. Bary, A treatise on trigonometric series, Vol. I, Pergamon Press, New York and Oxford, 1964.

2. J. B. Garnett, Bounded analytic functions, Academic Press, New York, 1981.

3. Ch. Pommerenke, Univalent functions, Vandenhoeck \& Ruprecht, Göttingen, 1975.

Department of Mathematics and Statistics, University of Auckland, Auckland, New Zealand 